\title{
Asymptotic solution of ultrasonic near-field levitation problem
}

\author{
I. F. Melikhov \\ ITMO University, Kronverkskiy, 49, St. Petersburg, 197101, Russia \\ ivan.melikhov@gmail.com
}

PACS 43.25.Uv

DOI 10.17586/2220-8054-2017-8-2-260-265

\begin{abstract}
Ultrasonic near-field levitation allows suspension of a moderately large object at a height of tens of microns above sound actuator. We developed an asymptotic approach to describe the air dynamics in the gap between an acoustic source and the levitating object. The suggested method allows computation of the lifting force. Due to resolving of both viscous and inertial effects, it remains applicable across a wide range of levitation distances. The paper explains theoretical background of the model and presents a numerical solution of the obtained equations. The results are compared to published numerical and experimental data showing very good agreement.
\end{abstract}

Keywords: ultrasonic levitation, acoustic streaming, asymptotic analysis.

Received: 13 January 2017

Revised: 19 January 2017

\section{Introduction}

This work focuses on a specific kind of acoustic levitation, which is called ultrasonic near-field levitation. This name comes from the observed small levitation heights: from $5 \mathrm{um}$ to $500 \mathrm{um}$. The phenomenon is employed in contactless transportation systems. The suspension of an object at extremely small distance was reported in 1964 by Salbu [1]. The first implementation of this technology for transport systems was utilized soon thereafter. A system based on flexural traveling waves of a guideline were studied in [2,3]. The works [4,5] suggested another approach: they used a trolley with ultrasonic source.

The theoretical base of acoustic levitation was founded by Lord Rayleigh [6,7], who considered acoustic pressure in inviscid fluid. His theory was finally finished much later in the papers [8,9]. This approach was used for lifting force estimation in the works [2,3]. However, this method is not applicable for small levitation distances. The reason is that for the heights comparable with acoustic boundary layer thickness, viscous effects begin to play a larger role. There is a plenty of purely viscous approaches. The absence of inertia terms is beneficial in terms of analysis. In some particular cases, it allows exact analytical description of flow in a domain with distributed velocity on the border [10]. However, the most wide-spread method is based on lubrication theory. In particular, this theory was used in the works [4,5] for lifting force estimation. The papers [12-14] also discuss this matter in greater detail. Unfortunately, the lubrication theory is limited to very small levitation heights.

Direct numerical simulation is more accurate. There are many computational methods for wave propagation in a medium (i.e. one is based on the cellular automation rules [11]). However, the most studied are mesh-based methods, such as finite differences or finite element methods. The paper [15] suggests a numerical scheme which gives results in good agreement with those obtained experimentally. A similar study was carried out in [16]. These papers also show that simplified approaches, e.g. purely acoustic and purely viscous, are much less accurate than numerical solution; however, the latter requires enormous computation resources.

The present paper suggests a new semi-analytical approach which allows one to resolve both inertial (acoustic) and viscous effects. The implemented model covers a wide range of levitation distances but remains computationally easy.

The next section describes the problem and gives basic equations of fluid dynamics. In section 3 , the governing equations are derived. We sequentially carry out scaling analysis, asymptotic analysis and Fourier analysis. The results are discussed in the section 4 , and then are summed up in conclusion.

\section{Problem statement}

We study the levitation of a rigid disk of radius $R$, as shown in the Fig. 1. Consider a flat ultrasonic transducer which vibrates with amplitude $a$ and circular frequency $\omega$. Displacement of the radiating face is described by:

$$
H_{s}(t)=a \cos (\omega t) \text {. }
$$

The levitating disk is assumed to be motionless and floating at the levitation height $H_{0}$. Then, the gap thickness is given by:

$$
h(t)=H_{0}-H_{s}(t) .
$$




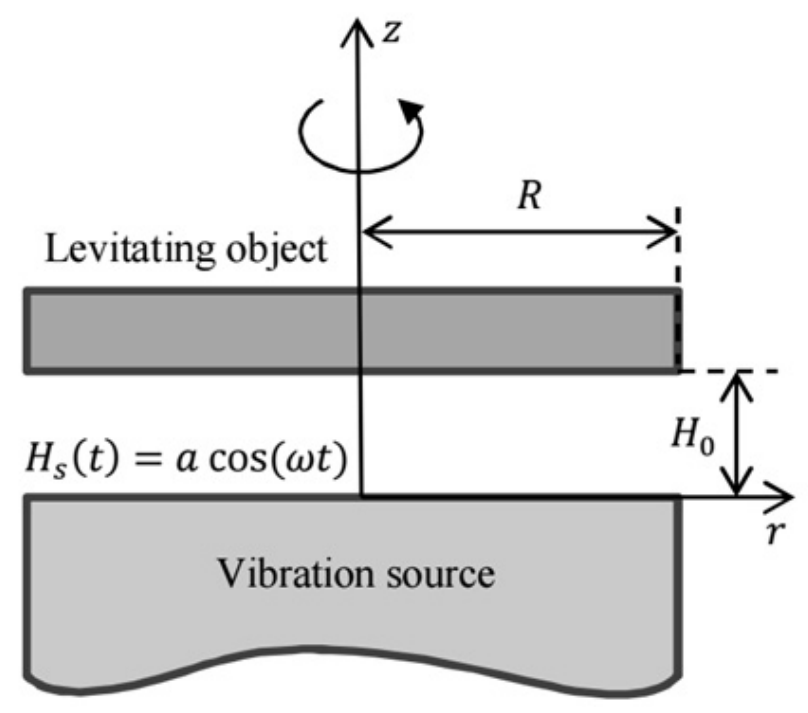

FIG. 1. Levitation of a disk

We start with basic equations of fluid dynamics (e.g. see [17]). First, we consider Navier-Stokes equations for a compressible fluid:

$$
\begin{gathered}
\rho\left(\partial_{t} \mathbf{v}+(\mathbf{v} \cdot \nabla) \mathbf{v}\right)=\nabla \cdot(-p \mathbf{I}+\tau), \\
\partial_{t} \rho+\nabla \cdot(\rho \mathbf{v})=0,
\end{gathered}
$$

where $\rho$ is the gas density, $\mathbf{v}=\left(v_{r}, v_{z}\right)$ is the velocity vector, $p$ is the pressure, $\mathbf{I}$ is the unit tensor, and $\tau$ is the viscous stress tensor given by:

$$
\tau=\mu\left[\nabla \mathbf{v}+(\nabla \mathbf{v})^{T}\right]+\left(\mu^{b}-\frac{2}{3} \mu\right)(\nabla \cdot \mathbf{v}) \mathbf{I},
$$

with $\mu$ for the dynamic viscosity of gas, and $\mu^{b}$ for the bulk viscosity.

The dynamic equations have to be accompanied by the energy equation:

$$
\rho T\left(\partial_{t} s+\mathbf{v} \cdot \nabla s\right)=\nabla \cdot(k \nabla T)+\Phi,
$$

where $T$ is the temperature, $s$ is the gas entropy, $k$ is the thermal conductivity of the gas, and $\Phi$ describes viscous dissipation of energy:

$$
\Phi=\tau_{j k} \frac{\partial v_{i}}{\partial x_{k}} .
$$

In addition, the viscosity-temperature dependence is given by Sutherland's law [18]:

$$
\mu=\mu_{0}\left(\frac{T}{T_{0}}\right)^{3 / 2} \frac{T_{0}+C}{T+C}
$$

where $\mu_{0}, T_{0}, C$ are empirical constants.

Finally, we assume that the pressure, density and temperature are connected by the ideal gas law:

$$
p=\rho \frac{\mathcal{R}}{\mathcal{M}} T,
$$

where $\mathcal{R}$ is the universal gas constant, and $\mathcal{M}$ is the molar mass of the gas.

The lifting force is computed by integration of $\sigma_{z z}$ over the disk's surface:

$$
F=-\iint\left(\left.\sigma_{z z}\right|_{z=H}\right) d x_{1} d x_{2},
$$

where

$$
\sigma_{z z}=-p+\mu\left[2 \frac{\partial v_{z}}{\partial z}+\left(\mu^{b}-\frac{2}{3} \mu\right) \nabla \cdot \mathbf{v}\right]
$$




\section{Governing equations}

The general equations may be significantly simplified in a few steps. First, scaling analysis is performed. Doing so allows us to neglect some terms in the equations. Then, the dimension of the continuity equation is reduced by averaging it through the thickness. After that, we introduce a small parameter, the ratio of the vibration amplitude $a$ to the gap thickness $H_{0}$, and seek solution in the form of an asymptotic series. Finally, we focus on the stationary part of the solution, which creates time-independent lifting force. In addition, non-reflective boundary conditions are developed on the gap's edge.

\subsection{Scaling analysis}

We introduce dimensionless variables:

$$
\begin{array}{lllll}
\tilde{r}=\frac{r}{R}, & \tilde{z}=\frac{z}{H_{0}}, & \tilde{t}=\omega t, & \tilde{v}=\frac{v_{z}}{\omega H_{0}}, & \tilde{u}=\frac{v_{r}}{\omega R}, \\
\tilde{p}=\frac{p}{p_{0}}, & \tilde{\rho}=\frac{\rho}{\rho_{0}}, & \tilde{\mu}=\frac{\mu}{\mu_{0}}, & \tilde{s}=\frac{s}{c_{v}}, & \tilde{T}=\frac{T}{T_{0}},
\end{array}
$$

where $c_{v}$ is the specific heat capacity at constant volume, and $p_{0}, \rho_{0}, \mu_{0}, T_{0}$ are pressure, density, viscosity, and temperature at normal conditions.

The developed theory relies on the following assumptions:

- the gap thickness is much smaller than the acoustic wavelength;

- the gap thickness is much smaller than its length;

- the vibration amplitude is much smaller than the gap thickness.

For ultrasonic near-field levitation, the typical gap thickness is $20-400 \mathrm{um}$, acoustic wavelength at $20 \mathrm{kHz}$ is about $2 \mathrm{~cm}$, and the disk radius is $1-10 \mathrm{~cm}$; the vibration amplitude varies in the range 1-10 um. Therefore, the first two assumptions always hold. The third one will be used to introduce a small parameter.

The given assumptions allow significant simplification of the governing equations. First, the energy equation (5) is reduced to the adiabatic relation:

$$
\tilde{\rho}=\tilde{p}^{1 / \gamma} .
$$

Using the ideal gas law (7) and viscosity-temperature dependence (6), one expresses viscosity in terms of pressure.

The dynamic equations (3a)-(3b) take simpler form as well:

$$
\begin{gathered}
\partial_{\tilde{z}} \tilde{p}=0, \\
\gamma K^{2} \tilde{\rho}\left(\partial_{\tilde{t}} u+\left(\tilde{u} \partial_{\tilde{r}} \tilde{u}+\tilde{v} \partial_{\tilde{z}} \tilde{u}\right)\right)=-\partial_{\tilde{r}} \tilde{p}+\Sigma \partial_{\tilde{z}}\left(\tilde{\mu} \partial_{\tilde{z}} \tilde{u}\right), \\
\partial_{\tilde{t} \tilde{\rho}+\tilde{\nabla} \cdot(\tilde{\rho} \tilde{\mathbf{v}})=0,}
\end{gathered}
$$

where $K^{2}=\omega^{2} R^{2} \gamma^{-1} \rho_{0} / p_{0}$ is the squared dimensionless acoustic wavenumber, and $\Sigma=\mu_{0} \omega R^{2} /\left(p_{0} H_{0}^{2}\right)$ is the so-called squeeze number.

The equation (11a) implies constant pressure across the gap. Together with (10), (7), (6) it results in $z$ independence of density, temperature, and viscosity.

There are no-slip boundary conditions on the sound source's and disk's surfaces:

$$
\begin{gathered}
\left.\tilde{u}\right|_{\tilde{z}=\tilde{H}_{s}}=\left.\tilde{u}\right|_{\tilde{z}=1}=0, \\
\left.\tilde{v}\right|_{\tilde{z}=\tilde{H}_{s}}=-\partial_{\hat{t}} \tilde{h}=-a / H_{0} \sin (\tilde{t}),\left.\quad \tilde{v}\right|_{\tilde{z}=\tilde{H}}=0 .
\end{gathered}
$$

Further, we will omit tilde over the dimensionless variables.

In addition, it is convenient to average the continuity equation (11c) across the gap:

$$
\frac{\partial}{\partial t}(\rho h)+\frac{1}{r} \frac{\partial}{\partial r}(r \rho h \bar{u})=0,
$$

where the gap-averaged velocity is:

$$
\bar{u}=\frac{1}{h} \int_{H_{s}}^{1} u d z .
$$




\subsection{Asymptotic analysis}

We introduce the small parameter $\varepsilon=a / H_{0}$ - ratio of the vibration amplitude to the average gap thickness. The solution is sought in the form of asymptotic series:

$$
\begin{array}{cc}
\rho=1+\varepsilon \rho^{(1)}+\varepsilon^{2} \rho^{(2)}+O\left(\varepsilon^{3}\right), & p=1+\varepsilon p^{(1)}+\varepsilon^{2} p^{(2)}+O\left(\varepsilon^{3}\right), \\
\mu=1+\varepsilon \mu^{(1)}+\varepsilon^{2} \mu^{(2)}+O\left(\varepsilon^{3}\right), & u=0+\varepsilon u^{(1)}+\varepsilon^{2} u^{(2)}+O\left(\varepsilon^{3}\right), \\
v=0+\varepsilon v^{(1)}+\varepsilon^{2} v^{(2)}+O\left(\varepsilon^{3}\right), \\
h=1+\varepsilon h^{(1)}(t), \quad h^{(1)}(t)=-\cos (t) .
\end{array}
$$

The equations (10) and (6) give expressions for the density terms:

$$
\rho^{(1)}=\frac{1}{\gamma} p^{(1)}, \quad \rho^{(2)}=\frac{1}{\gamma} p^{(2)}-\frac{\gamma-1}{2 \gamma^{2}}\left(p^{(1)}\right)^{2},
$$

and the viscosity term:

$$
\mu^{(1)}=\frac{(\gamma-1)}{2 \gamma} \frac{T_{0}+3 C}{T_{0}+C} p^{(1)}=M p^{(1)} .
$$

Since we focus on the steady levitation process, it is natural to look for solution in the form of the Fourier series:

$$
\begin{gathered}
p^{(m)}=\sum_{n=1}^{\infty} p_{n}^{(m)} e^{\mathrm{i} n t}, \quad u^{(m)}=\sum_{n=1}^{\infty} u_{n}^{(m)} e^{\mathrm{i} n t}, \\
h^{(1)}=\sum_{n=1}^{\infty} h_{n}^{(1)} e^{\mathrm{i} n t}=-\cos (t)=-\frac{1}{2}\left(e^{\mathrm{i} t}+e^{-\mathrm{i} t}\right) .
\end{gathered}
$$

Substituting these expressions into the equations above we get the system on the coefficient of the Fourier series (17). The first order gives non-trivial solution only for the terms with numbers $n= \pm 1$. However, the timeaveraged lifting force is zero. Therefore, a second-order solution is required. In order to compute the time-averaged lifting force, it is enough to study only zero-harmonic solution of the second-order problem.

The final governing equations which describe fluid flow in the gap are listed below. First-order continuity equation has the form:

$$
\frac{\mathrm{i}}{\gamma} p_{1}^{(1)}+\frac{1}{r} \frac{\partial}{\partial r}(r \bar{u})=\frac{\mathrm{i}}{2} .
$$

It is equipped with non-reflective boundary condition on pressure on the edge of the gap. This condition allows propagation of outgoing acoustic waves outside the gap:

$$
\left.\partial_{r} p_{1}^{(1)}\right|_{r=1}=\mathbf{i} K p_{1}^{(1)}
$$

The momentum equation with its boundary conditions are written as:

$$
\begin{gathered}
\mathrm{i} \gamma K^{2} u_{1}^{(1)}=-\partial_{r} p_{1}^{(1)}+\Sigma \partial_{z z}^{2} u_{1}^{(1)}, \\
\left.u_{1}^{(1)}\right|_{z=0}=0,\left.\quad u_{1}^{(1)}\right|_{z=1}=0 \\
\bar{u}_{1}^{(1)}=\int_{0}^{1} u_{1}^{(1)} d z
\end{gathered}
$$

and the transversal velocity is given by:

$$
v_{1}^{(1)}=\frac{\mathrm{i}}{2}-\int_{0}^{z}\left(\frac{\mathrm{i}}{\gamma} p_{1}^{(1)}+\frac{1}{r} \frac{\partial}{\partial r}\left(r u_{1}^{(1)}\right)\right) d z^{\prime} .
$$

The second-order equations for time-averaged (zero harmonic) values are the following. The continuity equation with the pressure boundary condition are

$$
\begin{gathered}
\frac{1}{r} \frac{\partial}{\partial r}\left(r \bar{u}_{0}^{(2)}+r\left[\left(p^{(1)} / \gamma-h^{(1)}\right) \bar{u}^{(1)}\right]_{0}\right)=0, \\
\left.p_{0}^{(2)}\right|_{r=1}=0
\end{gathered}
$$


and velocity can be found from:

$$
\begin{gathered}
\mathrm{i} K^{2}\left[n p_{n}^{(1)} u_{-n}^{(1)}\right]_{0}+\gamma K^{2}\left[u^{(1)} \partial_{r} u^{(1)}+v^{(1)} \partial_{z} u^{(1)}\right]_{0}=-\partial_{r} p_{0}^{(2)}+\Sigma\left(\partial_{z z}^{2} u_{0}^{(2)}+M\left[p^{(1)} \partial_{z z}^{2} u^{(1)}\right]_{0}\right), \\
\left.u_{0}^{(2)}\right|_{z=0}=\left[h^{(1)} \partial_{z} u^{(1)}\right]_{0},\left.\quad u_{0}^{(2)}\right|_{z=1}=0 \\
\bar{u}_{0}^{(2)}=\int_{0}^{1} u_{0}^{(2)} d z-\left[h^{(1)} \bar{u}^{(1)}\right]_{0}
\end{gathered}
$$

where $[\cdot]_{0}$ denotes zero harmonic of the expression (e.g. $\left[\left(p^{(1)}\right)^{2}\right]_{0}=p_{1}^{(1)} p_{-1}^{(1)}+p_{1}^{(-1)} p_{1}^{(1)}$ ).

The first-order equations (19), (21a) with the boundary conditions (20), (21b) are solved first. Then, one can calculate the transversal velocity (22). Substituting the first-order solution into the second-order equations: (23a), (24a) with the boundary conditions (23b), (24b), one obtains the time-averaged pressure.

Finally, the total lifting force can be calculated as:

$$
F_{0}=-\left.2 \pi \int \sigma_{z z}\right|_{z=1} r d r=2 \pi \int p_{0}^{(2)} r d r .
$$

\section{Results and discussion}

The governing equations show that there are two mechanisms of ultrasonic near-field levitation: inertial (acoustic) and viscous. Their intensity is described by the wavenumber $K$ and squeeze number $\Sigma$ respectively. The ratio of $\gamma K^{2}$ to $\Sigma$, which is included into equations (21a), (24a), is proportional to squared ratio of the gap thickness to the acoustic boundary layer thickness:

$$
\Pi=\frac{\gamma K^{2}}{\Sigma}=\frac{H_{0}^{2} \omega \rho_{0}}{\mu_{0}}=2\left(\frac{H_{0}}{\delta}\right)^{2},
$$

where $\delta=\sqrt{2 \mu_{0} /\left(\omega \rho_{0}\right)}$ is the boundary layer thickness [17]. Its value is $\delta \approx 15 \mathrm{um}$ in the case of air and $20 \mathrm{kHz}$ vibration frequency. When the gap is large, $\Pi \gg 1$, inertial effects are more important and acoustic models can be used; otherwise, when the gap is smaller than boundary layer, $\Pi \ll 1$, viscous effects dominate and viscous models are applicable. For intermediate regimes, $\Pi \approx 1$, both effects are important, and the presented model resolves them.

The obtained equaitons were solved by FEM in COMSOL Multiphysics software.

Figure 2 shows the comparison of the presented approach with a full numerical simulation and experimental data from the work [15]. The agreement is very good. However, our model requires the solution of 5 linear time-independent PDEs which is much easier computationally than direct simulation based on the general fluid dynamics equations.
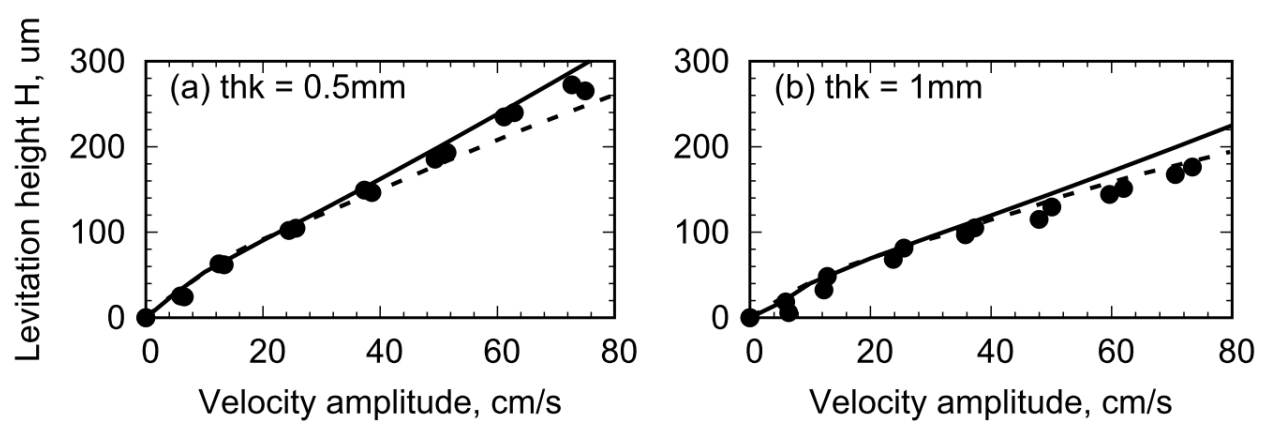

FIG. 2. Levitation height as a function of velocity of vibrating face. Disk radius $20 \mathrm{~mm}$, disk thickness $d=0.5-1 \mathrm{~mm}$, frequency $19.5 \mathrm{kHz}$. Solid lines denote the presented model; dashed line corresponds to numerical results of [15]; dots represent experimental data from [15] 


\section{Conclusion}

We presented a new approach for modeling of ultrasonic near-field levitation. In comparison to existing analytical works, our method covers a wide range of levitation distances and resolves both viscous and acoustic effects. However, it remains computationally simple in contrast to straightforward numerical simulation.

Our approach allows us to distinguish three regimes of levitation: viscous one for small levitation heights; acoustic one for large levitation distances; and intermediate visco-acoustic for moderate heights.

The model was successfully validated against published modeling and experimental results.

\section{Acknowledgements}

This work was partially financially supported by the Government of the Russian Federation (grant 074-U01), by grant MK-5161.2016.1 of the President of the Russian Federation, by grant 16-11-10330 of Russian Science Foundation.

\section{References}

[1] Salbu E.O.J. Compressible Squeeze Films and Squeeze Bearings. Journal of Basic Engineering, ASME, 1964, 86, P. 355-364.

[2] Hashimoto Y., Koike Y., Ueha S. Near-field acoustic levitation of planar specimens using flexural vibration. The Journal of the Acoustical Society of America, 1996, 100, P. 2057-2061.

[3] Hashimoto Y., Koike Y., Ueha S. Transporting objects without contact using flexural traveling waves. The Journal of the Acoustical Society of America, 1998, 103, P. 3230-3233.

[4] Yoshimoto S., Anno Y., Sato Y., Hamanaka K. Float Characteristics of Squeeze-Film Gas Bearing with Elastic Hinges for Linear Motion Guide. JSME International Journal Series C, 1997, 40, P. 353-359.

[5] Yoshimoto S., Kobayashi H., Miyatake M. Float characteristics of a squeeze-film air bearing for a linear motion guide using ultrasonic vibration. Tribology International, 2007, 40, P. 503-511.

[6] Lord Rayleigh. On the pressure of vibrations Philosophical. Magazine Series 6, 1902, 3, P. $338-346$.

[7] Lord Rayleigh. On the momentum and pressure of gaseous vibrations, and on the connexion with the virial theorem. Magazine Series 6, 1905, 10, P. 364-374.

[8] Chu B.-T., Apfel R.E. Acoustic radiation pressure produced by a beam of sound. The Journal of the Acoustical Society of America, 1982, 72, P. $1673-1687$.

[9] Lee C.P., Wang T.G. Acoustic radiation pressure. The Journal of the Acoustical Society of America, 1993, 94, P. 1099-1109.

[10] Makeev I.V, Popov I.Yu. Steady Stokes flow between confocal semi-ellipses. Nanosystems: Phys. Chem. Math., 2016,7 (2), P. $324-331$.

[11] Yamazaki Y, Ohmori S. Ultradiscretization of reaction-diffusion type partial differential equations exhibiting pulse propagation. Nanosystems: Phys. Chem. Math., 2017, 8 (1), P. 38-41.

[12] Minikes A., Bucher I. Coupled dynamics of a squeeze-film levitated mass and a vibrating piezoelectric disc: numerical analysis and experimental study. Journal of Sound and Vibration, 2003, 263, P. 241-268.

[13] Minikes A., Bucher I., Haber S. Levitation force induced by pressure radiation in gas squeeze films. The Journal of the Acoustical Society of America, 2004, 116, P. 217-226.

[14] Ilssar D., Bucher I. On the slow dynamics of near-field acoustically levitated objects under High excitation frequencies. Journal of Sound and Vibration, 2015, 354, P. 154-166.

[15] Nomura H., Kamakura T., Matsuda K. Theoretical and experimental examination of near-field acoustic levitation. The Journal of the Acoustical Society of America, 2002, 111, P. 1578-1583.

[16] Minikes A., Bucher I. Comparing numerical and analytical solutions for squeeze-film levitation force. Journal of Fluids and Structures, 2006, 22, P. 713-719.

[17] Landau L.D., Lifshitz E.M. Fluid Mechanics, 2nd ed., Vol. 6, Pergamon Press, Oxford, 1987, 532 p.

[18] Sutherland W. The viscosity of gases and molecular force. Philosophical Magazine Series 5, 1893, 36, P. $507-531$. 